with the form of craters produced by simulated micrometeorites, these particles having been accelerated using a Van der Graff accelerator to known velocities in the 1 to $20 \mathrm{~km} \mathrm{~s}^{-1}$ range. In many cases these characteristics indicated the relatively low impact velocity of about $7 \mathrm{~km} \mathrm{~s}^{-1}$. So both the curves in the figure have to be moved to the right by factors varying with the velocity dependence of microphone response, penetration hole size and crater diameter. As these effects are functions of meteoroid momentum or kinetic energy the use of this new velocity determination is still not sufficient to bring the curves together.

Two other things are assumed when dealing with microcraters, first that the ratio of the diameters of the microcrater pit and the impacting particle $\eta$ is 2 and second that the density of the particle is $3 \mathrm{~g} \mathrm{~cm}^{-3}$. Both these assumptions need to be reconsidered; recent laboratory experiments have shown that $\eta$ decreases with particle density and also that the mean particle density varies with mass. Both these factors make the interpretation of microcraters more difficult. Measurements of crater circularity have led Brownlee (University of Washington) et al. (4th Lunar Science Conference, Houston, 1973), to the conclusion that the majority of microparticles have equidimensional shapes and that shallow craters are produced by oblique incidence so the direction of the incident flux has also to be taken into account.

The main problem however lies in estimating the time the rocks under consideration have remained exposed on the lunar surface. One way of doing this is to count the tracks in the rock surface produced by retarding solar flare particles. Knowing the present day flare flux and assuming that solar activity has remained constant for about $10^{6}$ years gives the exposure age. This second assumption is the stumbling block; the data of Neukum and Schneider could indicate that either a lower particle flux existed in the past or

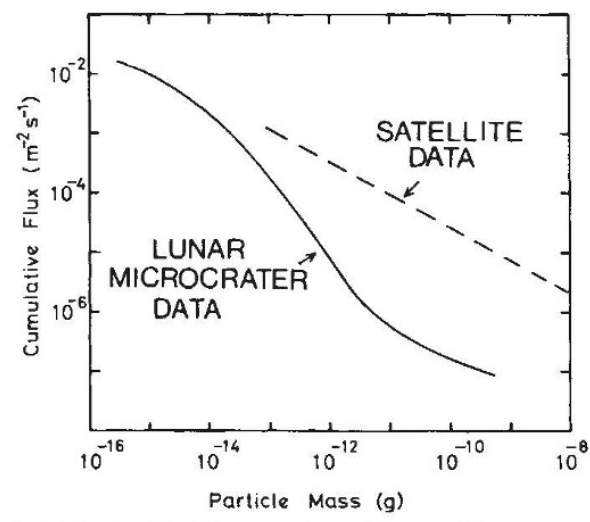

Number of micrometeoroids, with mass greater than the given mass, which will impact every second on a square metre of exposed. surface one astronomical unit from the Sun. that the solar flare flux was higher then than now-both would produce the same result.

McDonnell, Ashworth, Flavill, Bateman and Jennison (University of Kent) overcome this problem by considering surfaces with an equilibrium distribution of craters. Laboratory simulation indicates that solar wind sputter erosion $\left(0.043 \AA \mathrm{yr}^{-1}\right)$ is the dominant factor determining crater lifetime. Knowing this erosion rate enables the microparticle influx to be derived from the equilibrium distribution. The authors conclude (COSPAR, Sao Paulo, Brazil) that the microparticle influx has increased by a factor of four in the last $10^{5}$ years.

This would account for the discrepancy shown in the figure but creates the question of why this flux has increased.

\section{Adaptations of plants to drought}

\section{from Peter D. Moore}

THE $C_{4}$ dicarboxyl acid pathway of carbon fixation has now been recognised in a wide variety of plant species from at least six orders of flowering plants. In this process $\mathrm{CO}_{2}$ combines with phosphoenolpyruvate and is transported to the bundle sheath cells in the form of dicarboxylic acids, where it is released as $\mathrm{CO}_{2}$ to be fixed by the conventional Calvin $\left(\mathrm{C}_{3}\right)$ system. The widespread occurrence of the mechanism suggests that it arose independently in more than one order (Evans in Photosynthesis and Photorespiration, edit. by Hatch, Osmond and Slatyer, 130, Wiley, New York; 1971). This being so, it has been difficult to study the evolutionary development of the process. Yet the work of Bjorkman et al. (Yb. Carnegie Instn Wash., 68, $620 ; 1969$ and 69,$640 ; 1970$ ) indicates that very few genes are involved.

Troughton (in Photosynthesis and Photorespiration, 124; 1971) has proposed that a study of the isotopic ratio of ${ }^{13} \mathrm{C}$ to ${ }^{12} \mathrm{C}$ (this is normally expressed in relation to a standard and is termed $\delta^{13} \mathrm{C}$ ) in plant tissue should indicate the relative importance of the two systems of carbon fixation at the time when the plant was alive. This is because $C_{3}$ plants discriminate more effectively against ${ }^{13} \mathrm{C}$ in their carbon uptake than do $C_{4}$ plants; as a result $C_{3}$ plant tissue has an average $\delta^{13} \mathrm{C}$ of $-29 \%$ (that is proportionately less ${ }^{13} \mathrm{C}$ than in the air) whereas $\mathrm{C}_{4}$ plants' average ${ }^{13} \mathrm{C}$ is $-14 \%$.

Troughton, Wells and Mooney (Science, 185, 610; 1974) have now used this technique to study the relative importance of the $C_{4}$ pathway over the past 40,000 years in the $C_{4}$ desert plant Atriplex confertifolia. Two ancient middens of the pack rat Neotoma from the Mohave Desert region of Nevada yielded material of this species which dated from $>40,000$ yr (beyond the scope of radiocarbon determination) and $10,000 \mathrm{yr}$ respectively. The $\delta^{13} \mathrm{C}$ of the older sample is $-13.4 \%$ and that of the younger is $-16.2 \%$. A modern sample of $A$. confertifolia yielded a $\delta^{13} \mathrm{C}$ of $-14.8 \%$. Thus this species has been using the $C_{4}$ pathway for more than $40,000 \mathrm{yr}$.

Troughton et al. also determined the $\delta^{13} \mathrm{C}$ of Opuntia polyacantha tissues from the same sites. This species is a desert succulent which also concentrates carbon dioxide before fixing it by the conventional mechanism, but this time by crassulacean acid metabolism in which malic or citric acid concentrations build up during the night and the carbon accumulated in this way is subsequently used in the Calvin cycle. Once again these crassulacean acid (CAM) plants do not discriminate as strongly against ${ }^{13} \mathrm{C}$ as normal Calvin plants, and their $\delta^{13} \mathrm{C}$ values resemble those of $\mathrm{C}_{4}$ plants. In Opuntia polyacantha the $>40,000$-yr-old material gave a $\delta^{13} \mathrm{C}$ value of $-21.9 \%$, and the 10,000 -yr-old sample gave $-13.9 \%$. This suggests that the degree to which Opuntia depended upon the CAM technique of carbon concentration increased over the time interval between the two samples.

It is known that CAM plants become more dependent on night-time accumulation of carbon for their photosynthesis and growth under conditions of water stress. Indeed this mechanism can be regarded as an adaptation to dry habitat conditions in which normal daytime photosynthesis renders a plant liable to desiccation. The change in the $\delta^{13} \mathrm{C}$ values of the Opuntia tissue analysed may, therefore, reflect the changing contemporary climatic conditions in Nevada. Other plant remains found in the area support this suggestion, since they give evidence of pluvial juniper woodlands, corresponding to the Wisconsin glaciation of the north, giving way to arid desert at about 10,000 yr b.p.

There are similar indications from the Atriplex material. $\mathrm{C}_{4}$ plants generally and Atriplex confertifolia in particular are frequently associated with rather dry habitats; their cellular resistance to $\mathrm{CO}_{2}$ exchange is apparently lower than in $\mathrm{C}_{3}$ plants, which may account indirectly for their capacity to withstand drought. It is significant, therefore, that the remains of Atriplex are considerably more abundant in the 10,000 -yr-old specimen than in the 40,000 -yr-old one. Thus palaeontological and palaeobiochemical evidence provide complementary information concerning the climatic changes during this period. 